\title{
Remarks on the Breakdown of Smooth Solutions for the 3-D Euler Equations
}

\author{
J. T.Beale ${ }^{1 \star}$, T. Kato ${ }^{2 \star \star}$, and A. Majda ${ }^{2 \star \star \star}$ \\ 1 Department of Mathematics, Duke University, Durham, NC 27701, USA \\ 2 Department of Mathematics, University of California, Berkeley, CA 94720, USA
}

\begin{abstract}
The authors prove that the maximum norm of the vorticity controls the breakdown of smooth solutions of the 3-D Euler equations. In other words, if a solution of the Euler equations is initally smooth and loses its regularity at some later time, then the maximum vorticity necessarily grows without bound as the critical time approaches; equivalently, if the vorticity remains bounded, a smooth solution persists.
\end{abstract}

The motion of an ideal incompressible fluid is governed by a system of partial differential equations known as the Euler equations. For two-dimensional flow, solutions of the Euler equations with smooth initial data remain smooth for all time. However, in three space dimensions several numerical investigations $([2,3,11])$ predict very different phenomena. In particular, these computations suggest that solutions of the fluid equations which at first represent smooth flows may develop singularities, and furthermore that this breakdown of regularity signifies the onset of turbulent behavior. Qualitative arguments and numerical experiments indicate that the formation of singularities is related to the concentration of vorticity on successively smaller sets $([3,4])$. In this note we establish a mathematically rigorous link between the accumulation of vorticity and the formation of singularities for the 3-D Euler equations: we show that, if a solution is initially smooth and loses its regularity at some later time, then the maximum vorticity necessarily grows without bound as the critical time approaches. Therefore, it is not possible for other kinds of singularities (such as those in the deformation tensor or even milder singularities) to form before the vorticity becomes unbounded. In other words, the maximum norm of the vorticity alone controls the breakdown of smooth solutions for the 3-D Euler equations.

Euler's equations for the motion of an incompressible, inviscid fluid in free

\footnotetext{
^ Partially supported by O.N.R. Contract No. N00014-76-C-0316 and N.S.F. Grant No. MCS-81-01639

$\star \star$ Partially supported by N.S.F. Grant No. MCS-82-00171

$\star \star \star$ Partially supported by N.S.F. Grant No. MCS-81-02360
} 
space are known to have regular solutions for some time interval depending on a norm of a initial data. A concise treatment of the existence theory for these equations, as well as the Navier-Stokes equations of viscous flow, can be found in [6 or 5, Sect. 14]. In describing the solutions it is convenient to use the Sobolev space $H^{s}\left(\mathbb{R}^{3}\right)$, consisting of functions whose distributional derivatives up to order $s$ are in $L^{2}\left(\mathbb{R}^{3}\right), s$ being a positive integer; the norm of $u$ in $H^{s}$ is denoted by $|u|_{s}$.

Euler's equations of incompressible fluid motion are

$$
u_{t}+(u \cdot \nabla) u+\nabla p=0, \nabla \cdot u=0,
$$

where $u=u(x, t)$ is the velocity field and $p=p(x, t)$ is the pressure. The local existence theorem for Euler's equations can be stated as follows: Suppose an initial velocity field $u_{0}$ is specified in $H^{s}, s \geqq 3$, with $\left|u_{0}\right|_{3} \leqq N_{0}$, some $N_{0}>0$. Then there exists $T_{0}>0$, depending only on $N_{0}$, so that Eqs. (1) have a solution in the class

$$
u \in C\left([0, T] ; H^{s}\right) \cap C^{1}\left([0, T] ; H^{s-1}\right)
$$

at least for $T=T_{0}\left(N_{0}\right)$. (For the fact that $T_{0}$ depends only on $N_{0}$, see [10 or 13].) Of course, such a theorem gives no indication as to whether solutions actually lose their regularity or the manner in which they may do so. The property derived here is that, if the solution fails to be regular past a certain time, then the vorticity $\omega=\nabla \times \mathrm{u}$ must necessarily become unbounded.

Theorem. Let $u$ be a solution of Euler's equations as described above, and suppose there is a time $T_{*}$ such that the solution cannot be continued in the class (2) to $T=T_{*}$. Assume that $T_{*}$ is the first such time.

Then

$$
\int_{0}^{T_{*}}|\omega(t)|_{L^{\infty}} d t=\infty
$$

and in particular

$$
\limsup _{t \uparrow T_{*}}|\omega(t)|_{L^{\infty}}=\infty
$$

The proof of the Theorem which we give below also has the following immediate consequence:

Corollary. For some solution of Euler's equations, suppose there are constants $M_{0}$ and $T_{*}$ so that on any interval $[0, T]$ of existence of the solution in class (2), with $T<T_{*}^{*}$, the vorticity satisfies the a priori estimate

$$
\int_{0}^{T}|\omega(t)|_{L} \infty d t \leqq M_{0} .
$$

Then the solution can be continued in the class (2) to the interval $\left[0, T_{*}\right]$.

Similar statements hold for the Navier-Stokes equations; the arguments below apply equally well in this case.

We first claim that

$$
\limsup _{t \uparrow T_{*}}|u(t)|_{H^{s}}=\infty
$$


If not, then $|u(t)|_{s} \leqq C_{0}$ for some $C_{0}$ and all $t<T_{*}$. By the local existence theorem stated above, we can start a solution at any time $t_{1}$ with initial value $u\left(t_{1}\right)$, and this solution will be regular for $t_{1} \leqq t \leqq t_{1}+T_{0}\left(C_{0}\right)$, with $T_{0}$ independent of $t_{1}$. If $t_{1}>T_{*}-T_{0}$, we have then extended the original solution past time $T_{*}$, contrary to the choice of $T_{*}$.

To prove the theorem, we will assume

$$
\int_{0}^{T_{*}}|\omega(t)|_{L^{\infty}} d t \equiv M_{0}<\infty
$$

and show that

$$
|u(t)|_{H^{s}} \leqq C_{0}, t<T_{*},
$$

for some $C_{0}$, contradicting (3). Usually such bounds are obtained from inequalities which allow exponential growth. In this case our inequality leading to (5) will be slightly nonlinear but will still prevent arbitrarily large growth in a finite time.

We first estimate $\omega(t)$ in $L^{2}$. Taking the curl in (1) leads to the vorticity equation

We recall the important fact that

$$
\omega_{t}+u \cdot \nabla \omega=\omega \cdot \nabla u \text {. }
$$

$$
((u \cdot \nabla) w, w)=0
$$

at least for $w \in H^{1}$, where parentheses denote the inner product in $L^{2}=H^{0}$; this follows from integration by parts and the fact that $\nabla \cdot u=0$. Thus if we multiply (6) by $\omega$ and integrate, we have

$$
\frac{1}{2} \frac{d}{d t}|\omega|_{L^{2}}^{2}=(\omega \cdot \nabla u, \omega)
$$

The velocity $u$ is determined from $\omega$ by the relation

$$
u=-\nabla \times\left(\nabla^{-1} \omega\right) \text {. }
$$

Therefore the Fourier transforms of $\nabla u$ and $\omega$ satisfy $(\nabla u) \hat{(\xi)}=S(\xi) \hat{\omega}(\xi)$, where $S$ is a matrix which is bounded independent of $\xi$, and consequently $|\nabla u|_{L^{2}} \leqq C|\omega|_{L^{2}}$. Applying this to (8) gives

$$
\frac{d}{d t}|\omega|_{L^{2}}^{2} \leqq 2 C m(t)|\omega|_{L^{2}}^{2}
$$

where $m(t)=|\omega(t)|_{L^{\infty}}$, so that

or

$$
|\omega(t)|_{L^{2}} \leqq|\omega(0)|_{L^{2}} \exp C \int_{0}^{t} m(\tau) d t,
$$

with $M_{1}=\exp C M_{0}$.

$$
|\omega(t)|_{L^{2}} \leqq M_{1}|\omega(0)|_{L^{2}}
$$

Next we derive an energy estimate for (1) in terms of $|\nabla u|_{L^{\infty}}$. Let $\alpha$ be a multi-index with $|\alpha| \leqq s$, and let $v=D_{x}^{\alpha} u$. Applying $D_{x}^{\alpha}$ to (1), we have for $v$ the equation

$$
\mathrm{v}_{t}+u \cdot \nabla v+\nabla q=-F
$$


where $q=D^{\alpha} p$, and

$$
F=D^{\alpha}(u \cdot \nabla u)-u \cdot \nabla D^{\alpha} u .
$$

We will estimate $F$ using the calculus inequality

$$
\left|D^{\alpha}(f g)-f D^{\alpha} g\right|_{L^{2}} \leqq C\left(|f|_{H^{s}}|g|_{L^{\infty}}+|\nabla f|_{L^{\infty}}|g|_{H^{s-1}}\right)
$$

with $f=u$ and $g=\nabla u$. This inequality is well-known if $f \in H^{s} \cap C^{1}$ and $g \in H^{s} \cap C$ (see [8 or 12]), but we need it here with $g \in H^{s-1} \cap C$. The simple proof of (13) in the appendix of [8], based on the Gagliardo-Nirenberg inequalities, can be combined with a passage to the limit to show that it remains true in this case, even though the individual terms on the left in (13) may not be in $L^{2}$. Substituting in (13) we have $|F|_{L^{2}} \leqq C|\nabla u|_{L^{\infty}}|u|_{H^{s}}$.

We now proceed formally, multiplying (11) by $v$ and integrating to obtain

$$
\frac{1}{2} \frac{d}{d t}|v|_{0}^{2} \leqq\left. C|\nabla u|_{L} \infty u\right|_{s}|v|_{0} .
$$

We have used (7) and the orthogonality of gradients and vector fields of divergence zero. Summing over $\alpha$ with $0 \leqq|\alpha| \leqq s$, we now have

$$
\frac{d}{d t}|u|_{s}^{2} \leqq 2 C|\nabla u|_{L^{\infty}}|u|_{s}^{2}
$$

so that

$$
|u(t)|_{s} \leqq|u(0)|_{s} \exp \left(C \int_{0}^{t}|\nabla u(\tau)|_{L^{\infty}} d \tau\right) .
$$

The steps leading to (14) can be justified by regarding $v$ as the weak solution of a linear equation (11) in the class $C\left([0, T] ; L^{2}\right)$ and arguing as for linear hyperbolic equations. (We approximate $v(0)$ by smooth initial data, derive an $L^{2}$ energy estimate, and pass to the limit, using the uniqueness of weak solutions.) Weaker forms of (14) were derived earlier in $[10,13]$, and this sharper version was given in $[9$, pp. $62-63]$. $^{1}$

To complete the argument we use a time-independent estimate for $|\nabla u|_{L^{\infty}}$ in terms of bounds on $\omega$ and slight dependence on a higher norm of $u$,

$$
|\nabla u|_{L^{\infty}} \leqq C\left\{1+\left(1+\log ^{+}|u|_{3}\right)|\omega|_{L^{\infty}}+|\omega|_{L^{2}}\right\} .
$$

Here $C$ is a universal constant and $\log ^{+} a=\log a$ if $a \geqq 1, \log ^{+} a=0$ otherwise. This estimate is based on the relation (9) between $u$ and $\omega$. It was derived in the two-dimensional case by the second author in other work [7]. In view of (4) and (10), we can write (15) as

$$
|\nabla u|_{L^{\infty}} \leqq C\left\{1+m(t) \log \left(|u|_{3}+e\right)\right\} .
$$

Here and below, $C$, denotes a constant depending on $M_{0}$ and $T_{*}$.

1 Such estimates are valid for general symmetric hyperbolic systems - see [14] 
Now let $y(t)=|u(t)|_{s}+e$. Combining (14) and (16) we have

$$
y(t) \leqq y(0) \exp C \int_{0}^{t}\{1+m(\tau) \log y(\tau)\} d \tau,
$$

and if $z(t)=\log y(t)$,

$$
z(t) \leqq z(0)+C \int_{0}^{t}\{1+m(\tau) z(\tau)\} d \tau .
$$

It follows from Gronwall's inequality that $z(t)$ is bounded by a constant depending on $M_{0}, T_{*}$, and $\left|u_{0}\right|_{s}$, and (5) is established. The proof of the theorem is now completed except for the

Proof of (15). The relation between $\omega$ and $u$, as expressed in (9), is given explicitly by the Biot-Savart Law:

$$
u(x)=-\frac{1}{4 \pi} \int \frac{(x-y)}{|x-y|^{3}} x \omega(y) d y \equiv \int K(x, y) \omega(y) d y .
$$

We introduce a cut-off function $\zeta_{\rho}(x)$, satisfying $\zeta_{\rho}(x)=1$ for $|x|<\rho, \zeta_{\rho}(x)=0$ for $|x|>2 \rho$, and $\left|\nabla \zeta_{\rho}(x)\right| \leqq C / \rho$. Here $\rho \leqq 1$ is a radius to be chosen suitably small later on. We introduce a factor $\zeta_{\rho}(x-y)+\left[1-\zeta_{\rho}(x-y)\right]$ under the integral sign and split $\nabla u(x)$ into two terms, the first being $\nabla u^{(1)}(x)=\int \zeta_{\rho}(x-y) K(x-y) \nabla \omega(y) d y$. Since $|K(x-y)| \leqq C|x-y|^{-2}, K$, as a function of $y$, belongs to $L^{p}(\{y:|x-y|<2 \rho\})$ for $p<3 / 2$. For convenience we take $p=4 / 3$. By Hölder's inequality,

$$
\left|\nabla u^{(1)}(x)\right| \leqq|K|_{L^{4 / 3}}|\nabla \omega|_{L^{4}} \leqq C \rho^{1 / 4}|\nabla \omega|_{L^{4}},
$$

both norms being taken over $\{|x-y|<2 \rho\}$. By Sobolev's inequality

$$
|\nabla \omega|_{L^{4}} \leqq C|\nabla \omega|_{H^{1}} \leqq C|u|_{H^{3}},
$$

so that

$$
\left|\nabla u^{(1)}\right| \leqq C \rho^{1 / 4}|u|_{H^{3}}
$$

We are left with

$$
\nabla u(x)-\nabla u^{(1)}(x)=\int \nabla\left\{K(x-y)\left(1-\zeta_{\rho}(x-y)\right)\right\} \omega(y) d y \equiv \nabla u^{(2)}+\nabla u^{(3)},
$$

where $\nabla u^{(2)}$ is the integral over $\rho \leqq|x-y| \leqq 1$, and $\nabla u^{(3)}$ over $|x-y|>1$. For $\nabla u^{(2)}$ we estimate the two terms in the gradient separately and use

$$
|\nabla K(x-y)| \leqq C|x-y|^{-3}
$$

to obtain

$$
\left|\nabla u^{(2)}(x)\right| \leqq C\left\{\int_{\rho}^{1} r^{-3} \cdot r^{2} d r+\int_{\rho}^{2 \rho} r^{-2} \rho^{-1} r^{2} d r\right\}|\omega|_{L^{\infty}},
$$

or

$$
\left|\nabla u^{(2)}(x)\right| \leqq C(-\log \rho+1)|\omega|_{L^{\infty}} .
$$

Finally, (18) implies that $\nabla K$ is $L^{2}$ for $|x-y|>1$, and we can estimate $\nabla u^{(3)}$ by 
the Cauchy-Schwarz inequality

$$
\left|\nabla u^{(3)}(x)\right| \leqq C|\omega|_{L^{2}}
$$

Combining (17), (19), (20) we have

$$
|\nabla u|_{L^{\infty}} \leqq C\left\{\rho^{1 / 4}|u|_{3}+(1-\log \rho)|\omega|_{L^{\infty}}+|\omega|_{L^{2}}\right\} .
$$

If $|u|_{3} \leqq 1$, we take $\rho=1$; otherwise we choose $\rho$ so that the first term is 1 , i.e., $\rho=|u|_{3}^{-4}$, and (21) becomes

$$
|\nabla u|_{L^{\infty}} \leqq C\left\{1+\left(1+4 \log |u|_{3}\right)|\omega|_{L^{\infty}}+|\omega|_{L^{1}}\right\} .
$$

In either case (13) holds.

The inequality (15) is reminiscent of a somewhat different estimate in [1], which bounds a function in $L^{\infty}$ in terms of the $H^{n / 2}$-norm and the logarithm of a higher norm, $n$ being the space dimension.

Reemark. With only minor changes, the same proof applies to periodic fluid flow. In fact the proof of (15) is even simpler since the kernel expressing $u$ from $\omega$ has the same local behavior while the contribution from $\nabla u^{(3)}$ in (20) is absent in the periodic case. However, a more involved proof using additional ideas seems necessary for fluid flow in bounded domains.

\section{Bibliography}

1. Brezis, H., Gallouet, T.: Nonlinear Schrödinger evolution equations. J. Nonlinear Anal. 4, 677-681 (1980)

2. Chorin, A.: Estimates of intermittency, spectra, and blow-up in developed turbulence. Commun. Pure Appl. Math. 34, 853-866 (1981)

3. Chorin, A.: The evolution of a turbulent vortex. Commun. Math. Phys. 83, 517-535 (1982)

4. Frisch, U., Sulem, P. L., Nelkin, M.: A simple dynamical model of intermittent fully developed turbulence. J. Fluid Mech. 87, 719-736 (1978)

5. Kato, T.: Quasi-linear equations of evolution with applications to partial differential equations. In: Lecture Notes in Mathematics, Vol. 448, Berlin, Heidelberg, New York: Springer 25-70 (1975)

6. Kato, T.: Nonstationary flows of viscous and ideal fluids in $\mathbb{R}^{3}$. J. Funct. Anal. 9, 296-305 (1972)

7. Kato, T.: Remarks on the Euler and Navier-Stokes equations in $\mathbb{R}^{2}$. (to appear)

8. Klainerman, S., Majda, A.: Singular limits of quasilinear hyperbolic systems with large parameters and the incompressible limit of compressible fluids. Commun. Pure Appl. Math. 34, 481-524 (1981)

9. Majda, A.: Compressible Fluid Flow and Systems of Conservation Laws in Several Space Variables. In: Applied Math. Sciences Series, Vol. Berlin, Heidelberg, New York, Tokyo: Springer 1983

10. Marsden, J., Ebin, D., Fischer, A. E.: Diffeomorphism groups, hydrodynamics and relativity. Proceedings of the thirteenth biennial seminar of the Canadian Math. Congress, Vanstone, J. R. (ed.) Montreal, 1972

11. Morf, R., Orszag, S., Frisch, U.: Spontaneous singularity in three-dimensional incompressible flow. Phys. Rev. Lett. 44, 572-575 (1980)

12. Moser, J.: A rapidly convergent iteration method and nonlinear differential equations. Ann. Scuola Norm. Sup. Pisa 20, 265-315 (1966)

13. Temam, R.: Local existence of $C^{\infty}$ solutions of the Euler equations of incompressible perfect fluids. In: Lecture Notes in Mathematics, Vol. 565, Berlin, Heidelberg, New York: Springer 184-194 (1976)

14. Klainerman, S., Ponce, G.: Global, small amplitude solutions to nonlinear wave equations. Commun. Pure Appl. Math. 36, (1983)

Communicated by L. Nirenberg

Received September 20, 1983; in revised form December 7, 1983 